\title{
Propagation of Nonclassical Radiation through a Semiconductor Slab
}

\author{
D. Yu. Vasylyev, ${ }^{1,2}$ W. Vogel, ${ }^{1}$ T. Schmielau, ${ }^{3}$ K. Henneberger, ${ }^{4}$ and D.-G. Welsch ${ }^{5}$ \\ ${ }^{1}$ Arbeitsgruppe Quantenoptik, Institut für Physik, Universität Rostock, D-18051 Rostock, Germany \\ ${ }^{2}$ Bogolyubov Institute for Theoretical Physics, NAS of Ukraine, Metrolohichna 14-b, UA-03680 Kiev, Ukraine \\ ${ }^{3}$ Centre for Electronic Devices and Materials, Sheffield Hallam University, United Kingdom \\ ${ }^{4}$ Arbeitsgruppe Festkörpertheorie, Institut für Physik, Universität Rostock, D-18051 Rostock, Germany \\ ${ }^{5}$ Theoretisch-Physikalisches Institut, Friedrich-Schiller-Universität Jena, Max-Wien-Platz 1, D-07r43 Jena, Germany
}

\begin{abstract}
Based on a microscopic derivation of the emission spectra of a bulk semiconductor we arrive at a clear physical interpretation of the noise current operators in macroscopic quantum electrodynamics. This opens the possibility to study medium effects on nonclassical radiation propagating through an absorbing or amplifying semiconductor. As an example, the propagation of an incident squeezed vacuum is analyzed.

PACS numbers: 42.50.Nn, 78.20.-e, 42.50.Ct
\end{abstract}

The progress of experimental techniques has rendered it possible to almost completely measure the quantum statistics of radiation-matter systems, so that correlation functions up to, in principle, arbitrarily high orders can be detected (for a review of the various methods, see, e.g., Ref. [1]). Within the framework of quantum optics, the theoretical study of the interaction of light with complex material systems is typically based on simplifying model systems, effective-Hamiltonian schemes or related semi-phenomenological concepts rather than rigorous microscopic calculations [2].

On the other hand, in many-particle quantum theory, including semiconductor theory, much effort has been spent on the development of methods for microscopically describing complex material systems. This includes the description of coherent optical interactions by semiconductor Bloch equations and semiconductor luminescence equations as well as the development of nonequilibrium Green's function methods [3, 4]. In principle, these methods lead to infinite hierarchies of correlations, which are usually treated by properly developed methods of truncations and/or decorrelations.

It is well known that semiconductors can be used to generate nonclassical radiation [5]. In particular, the development of nano-structured systems has opened new possibilities of the generation and application of nonclassical radiation in integrated systems. For example, the correlated emission of single photons can be demonstrated by using quantum dots [6] and bound excitons in semiconductors [7]. Experiments with quantum wells [8] and quantum dots [9, 10, 11] also show the potential of semiconductors for the generation of entangled photons, which are of interest in quantum information processing.

In order to properly describe the generation and/or propagation of nonclassical radiation through complex material systems such as semiconductor slabs, one has simultaneously to deal with both higher-order radiationfield correlation functions and many-particle quantum statistics of the material system. Within the frame of macroscopic quantum electrodynamics (QED), methods of describing the quantized electromagnetic field in linearly responding (dispersing and absorbing/amplifying) media have been developed, with special emphasis on quantum-noise effects [12, 13, 14, 15, 16, 17. Based on given constitutive relations of the material system, field correlation functions of arbitrary order can be calculated in this way. On the other hand, methods of many-particle theory can be used to treat the radiation-matter interaction within the frame of microscopic QED, thereby calculating the relevant electromagnetic properties of the material system. Such methods have been used to study the propagation of radiation in semiconducting material, including amplification and lasing [19, 20], with the restriction to the intensity and the emission spectrum of the radiation, because of the elaborateness of the problem.

In the present paper we make the attempt to close the gap between macroscopic and microscopic QED, leading to a clear physical interpretation of the noise-current density introduced in macroscopic QED. In particular, appropriate combination of the results of the two methods offers the possibility to calculate arbitrary radiationfield correlation functions, as we demonstrate by studying the propagation of squeezed light through an absorbing/amplifying semiconductor slab.

We begin with the operator of the vector potential in Coulomb gauge, $\hat{\mathbf{A}}(\mathbf{r}, t)$, which obeys the inhomogeneous wave equation

$$
\square \hat{\mathbf{A}}(\mathbf{r}, t)=-\mu_{0} \hat{\mathbf{j}}(\mathbf{r}, t)
$$

and the equal-time commutation rule

$$
\left[\hat{\mathbf{A}}(\mathbf{r}, t), \frac{\partial}{\partial t} \hat{\mathbf{A}}\left(\mathbf{r}^{\prime}, t\right)\right]=\frac{i \hbar c}{\varepsilon_{0}} \overleftrightarrow{\delta}_{\mathrm{T}}\left(\mathbf{r}-\mathbf{r}^{\prime}\right)
$$

where $\overleftrightarrow{\delta}_{\mathrm{T}}(\mathbf{r})$ is the transverse tensorial delta function. The operator of the transverse current density, $\hat{\mathbf{j}}(\mathbf{r}, t)$, can be decomposed into the current density $\hat{\mathbf{j}}_{\text {med }}(\mathbf{r}, t)$ associated with the medium under consideration and an auxiliary, externally controlled $c$-number current density 
$\mathbf{j}_{\text {ext }}(\mathbf{r}, \mathbf{t})$ which is commonly set zero at the end of the calculations.

Starting from microscopic QED and employing Green's function (GF) techniques, for a dielectric medium in the steady state, the expectation value of the vector potential (in the Fourier domain) reads as

$$
\langle\hat{A}(\mathbf{r}, \omega)\rangle=-\mu_{0} \int d^{3} \mathbf{r}^{\prime} \overleftrightarrow{D}^{\mathrm{ret}}\left(\mathbf{r}, \mathbf{r}^{\prime}, \omega\right) \cdot \mathbf{j}_{\mathrm{ext}}\left(\mathbf{r}^{\prime}, \omega\right)
$$

where, in linear approximation, the inverse of the retarded photon GF $\overleftrightarrow{D}^{\text {ret }}\left(\mathbf{r}, \mathbf{r}^{\prime}, \omega\right)$ is given by

$$
\overleftrightarrow{D}^{\text {ret },-1}\left(\mathbf{r}, \mathbf{r}^{\prime}, \omega\right)=\square \delta\left(\mathbf{r}-\mathbf{r}^{\prime}\right)-\overleftrightarrow{P}^{\text {ret }}\left(\mathbf{r}, \mathbf{r}^{\prime}, \omega\right)
$$

with the retarded polarization tensor $\overleftrightarrow{P}^{\text {ret }}\left(\mathbf{r}, \mathbf{r}^{\prime}, \omega\right)$ being related to the complex (dielectric) susceptibility tensor $\overleftrightarrow{\chi}\left(\mathbf{r}, \mathbf{r}^{\prime}, \omega\right)=\overleftrightarrow{\chi}^{\prime}\left(\mathbf{r}, \mathbf{r}^{\prime}, \omega\right)+i \overleftrightarrow{\chi}^{\prime \prime}\left(\mathbf{r}, \mathbf{r}^{\prime}, \omega\right)$ of the medium according to

$$
\overleftrightarrow{P^{\mathrm{ret}}}\left(\mathbf{r}, \mathbf{r}^{\prime}, \omega\right)=-\frac{\omega^{2}}{c^{2}} \overleftrightarrow{\chi}\left(\mathbf{r}, \mathbf{r}^{\prime}, \omega\right)
$$

The Keldysh components of the photon GF,

$$
\begin{aligned}
\overleftrightarrow{D} & >\left(\mathbf{r}, \mathbf{r}^{\prime}, t-t^{\prime}\right)=\overleftrightarrow{D^{<}}<\left(\mathbf{r}^{\prime}, \mathbf{r}, t^{\prime}-t\right) \\
& =\frac{1}{i \hbar}\left[\left\langle\hat{\mathbf{A}}(\mathbf{r}, t) \hat{\mathbf{A}}\left(\mathbf{r}^{\prime}, t^{\prime}\right)\right\rangle-\langle\hat{\mathbf{A}}(\mathbf{r}, t)\rangle\left\langle\hat{\mathbf{A}}\left(\mathbf{r}^{\prime}, t^{\prime}\right)\right\rangle\right],
\end{aligned}
$$

describe field-field fluctuations. Solving the Dyson equation for these components, one obtains the optical theorem

$$
\begin{aligned}
D_{i j}^{\gtrless}\left(\mathbf{r}, \mathbf{r}^{\prime}, \omega\right)=\int & d^{3} \mathbf{r}_{1} \int d^{3} \mathbf{r}_{2} D_{i k}^{\mathrm{ret}}\left(\mathbf{r}, \mathbf{r}_{1}, \omega\right) \\
& \times P_{k l}^{\gtrless}\left(\mathbf{r}_{1}, \mathbf{r}_{2}, \omega\right) D_{l j}^{\mathrm{adv}}\left(\mathbf{r}_{2}, \mathbf{r}^{\prime}, \omega\right),
\end{aligned}
$$

where $P_{k l}^{\gtrless}$ are the corresponding Keldysh components of the polarization tensor, and summation over repeated indices is understood. Within the framework of macroscopic QED, Eq. (11) is regarded as being the operatorvalued inhomogeneous wave equation that corresponds to the Maxwell equations of the transverse part of the macroscopic electromagnetic field in a linear dielectric medium. Hence, $\hat{\mathbf{j}}_{\text {med }}(\mathbf{r}, \omega)$ must have the form

$$
\hat{\mathbf{j}}_{\text {med }}(\mathbf{r}, \omega)=-\varepsilon_{0} \omega^{2} \int d^{3} \mathbf{r}^{\prime} \overleftrightarrow{\chi}\left(\mathbf{r}, \mathbf{r}^{\prime}, \omega\right) \cdot \hat{\mathbf{A}}\left(\mathbf{r}^{\prime}, \omega\right)+\hat{\mathbf{j}}_{\mathrm{N}}(\mathbf{r}, \omega),
$$

and in place of Eq. (3) we obtain the operator-valued equation

$$
\begin{aligned}
& \hat{\mathbf{A}}(\mathbf{r}, \omega) \\
&=-\mu_{0} \int d^{3} \mathbf{r}^{\prime} \overleftrightarrow{D}^{\mathrm{ret}}\left(\mathbf{r}, \mathbf{r}^{\prime}, \omega\right) \cdot\left[\hat{\mathbf{j}}_{\mathrm{N}}\left(\mathbf{r}^{\prime}, \omega\right)+\mathbf{j}_{\mathrm{ext}}\left(\mathbf{r}^{\prime}, \omega\right)\right],
\end{aligned}
$$

where the noise current density operator $\hat{\mathbf{j}}_{\mathrm{N}}(\mathbf{r}, \omega)$ obeys the commutation relation [16]

$$
\left[\hat{\mathbf{j}}_{\mathrm{N}}(\mathbf{r}, \omega), \hat{\mathbf{j}}_{\mathrm{N}}^{\dagger}\left(\mathbf{r}^{\prime}, \omega^{\prime}\right)\right]=2 \varepsilon_{0} \hbar \omega^{2} \overleftrightarrow{\chi}^{\prime \prime}\left(\mathbf{r}, \mathbf{r}^{\prime} \omega\right) \delta\left(\omega-\omega^{\prime}\right)
$$

$\left[\hat{\mathbf{j}}_{\mathrm{N}}(\mathbf{r},-\omega)=\hat{\mathbf{j}}_{\mathrm{N}}^{\dagger}(\mathbf{r}, \omega)\right]$, which ensures the validity of Eq. (2).

With the analog of the optical theorem (7) in macroscopic QED, the relation

$$
\begin{aligned}
\overleftrightarrow{P}>\left(\mathbf{r}, \mathbf{r}^{\prime}, t-t^{\prime}\right) & =\overleftrightarrow{P}<\left(\mathbf{r}^{\prime}, \mathbf{r}, t^{\prime}-t\right) \\
& =\frac{\mu_{0}}{i \hbar}\left\langle\hat{\mathbf{j}}_{\mathrm{N}}(\mathbf{r}, t) \hat{\mathbf{j}}_{\mathrm{N}}\left(\mathbf{r}^{\prime}, t^{\prime}\right)\right\rangle
\end{aligned}
$$

can be derived. Moreover, using Green's function techniques, a Bethe-Salpeter equation between the polarization function and the correlation function of the medium current density can be derived [18]. In this way, the fluctuation of the noise current density in macroscopic QED can be directly related to the fluctuation of the microscopically well-defined and observable medium current density.

In the following we will deal with the propagation of TE-polarized radiation along the $x$ axis, through a semiconductor slab of thickness $L$ which is infinitely extended in the $y$-z-plane. For simplicity, we assume that the electric field is polarized along the $y$-axis $\hat{\mathbf{A}}=(0, \hat{A}, 0)$. Then, neglecting spatial dispersion, the complex refractive index inside the medium, $n=n^{\prime}+i n^{\prime \prime}$, is obtained from $n^{2}(\omega)=1+\chi(\omega)$.

Using Green's function technique, the spontaneous emission of the slab is studies in Ref. [19, 20]. A generalization of the results including spatial dispersion and providing exact relations between (spontaneous and stimulated) emission and linear absorption can be found in Ref. [22]. The intensity of the (spontaneously) emitted radiation can be given by

$$
I(\omega)=b(\omega) P(\omega) \hat{D}_{0}(\omega),
$$

where $\hat{D}_{0}$ is the vacuum-induced contribution to the photon spectral function, $P=2 i \operatorname{Im} P^{\text {ret }}$ is, according to (5), related to $\chi^{\prime \prime}$ associated with linear absorption/amplification, and $b$ is defined as the ratio between the recombination rate $P^{<}$of electron-hole pairs and $P$. The function $b(\omega)$ characterizes globally (i. e., inside and outside the slab) the emitted radiation and, as such, $b$ is accessible to direct observation in experiments. It generalizes Planck's formula for the black body radiation to nonequilibrium radiation of an excited medium in the steady state.

On the basis of Eq. (9), input-output relations can be derived, by introducing bosonic quasiparticle annihilation operators (normalization $\mathcal{N}_{ \pm}$)

$$
\hat{c}_{ \pm}(\omega)=\mathcal{N}_{ \pm}(\omega) \int_{-L / 2}^{L / 2} d x^{\prime}\left(e^{i n \omega x^{\prime} / c} \pm e^{-i n \omega x^{\prime} / c}\right) \hat{j}_{\mathrm{N}}\left(x^{\prime}, \omega\right)
$$

expressed in terms of the noise current density operator and associated with the slab-radiation excitations [13, 21]. On the other hand, the poles of $D^{\text {ret }}$ yield the polaritonic dispersion relations for the slab, which 
allows one to relate the operators $\hat{c}_{ \pm}$to the polaritonic annihilation operators 23]. Assuming that the input field is in the vacuum state, the intensity of the output field is obtained in just the same form as in Eq. (12), by identifying $\left\langle\hat{c}^{\dagger}(\omega) \hat{c}(\omega)\right\rangle \equiv b(\omega)$, where $\hat{c}(\omega)=\hat{c}_{+}(\omega)+\hat{c}_{-}(\omega)$.

Steadily excited semiconductors in quasi-equilibrium are of particular interest, such as exciton gases generated at low up to moderate excitation and light-emitting diodes working at high excitation. For quasi-equilibrium, due to the Kubo-Martin-Schwinger condition [24], the function $b(\omega)$ develops into a Bose distribution $b(\omega)=$ $(\exp [\beta(\hbar \omega-\mu)]-1)^{-1}$. The chemical potential $\mu$ starts at $\mu=0$ for complete thermal equilibrium and characterizes the degree of excitation beyond the thermal one for $\mu>0$. The crossover from absorption $\left(\chi^{\prime \prime}>0\right)$ to gain $\left(\chi^{\prime \prime}<0\right)$ appears at $\hbar \omega=\mu$. By expanding the product $b(\omega) \chi^{\prime \prime}(\omega)$ in Eq. (12) at $\hbar \omega=\mu$, it is seen that the spontaneous emission remains finite at the crossover, it is given by the slope of the absorption function $\chi^{\prime \prime}$. Since both $\chi^{\prime \prime}$ and $b$ switch their signs, the spontaneous emission stays positive in the whole frequency region, as it should be. Thus we obtain a unified description of absorption and amplification in semiconductors.

In order to demonstrate the usefulness of the established interrelation of microscopic and macroscopic QED, we consider the propagation of squeezed light through a semiconductor slab. Consider the squeezed vacuum state

$$
|\psi\rangle_{\mathrm{sv}}=\hat{S}|\psi\rangle_{\mathrm{v}},
$$

where the squeeze operator,

$$
\hat{S}=\exp \left\{\int_{0}^{\infty} d \omega\left[\xi^{*} \hat{a}\left(\omega_{0}+\omega\right) \hat{a}\left(\omega_{0}-\omega\right)-\text { h.c. }\right]\right\}
$$

acts on the multimode vacuum state $|\psi\rangle_{\mathrm{v}}$. The squeeze parameter is $\xi=|\xi| e^{i \phi_{\xi}}$, and $\hat{a}(\omega)$ are the photonic annihilation operators of the incident field on the left-hand side of the semiconductor slab. The input field on the right-hand side is assumed to be in the vacuum state.

Using Eqs (91), (12), and (14), the normally ordered squeezing spectrum $S_{\mathrm{sq}}=\int d \omega^{\prime} \omega_{0}^{2}\left\langle: \hat{A}(x, \omega) \hat{A}\left(x^{\prime}, \omega^{\prime}\right):\right\rangle_{\mathrm{sv}}$ is derived for $x=x^{\prime}=L$ as

$$
\begin{aligned}
S_{\mathrm{sq}} & =I(\omega)+\frac{\hbar \omega_{0}}{2 \pi \varepsilon_{0} c}\left\{|\mathcal{T}(\omega)|^{2} \sinh ^{2}|\xi|\right. \\
& \left.+e^{-2 i \omega_{0} L / c-i \phi_{\xi}}\left[\mathcal{T}^{*}(\omega)\right]^{2} \cosh |\xi| \sinh |\xi|+\text { c.c. }\right\} .
\end{aligned}
$$

Here we have assumed that the detector is placed on the right-hand side of the slab. The total transmission coefficient of the slab, $\mathcal{T}(\omega)$, reads in terms of the internal reflectivity $r(\omega)=[1-n(\omega)] /[1+n(\omega)] e^{i \omega n(\omega) L / c}$ as 25$]$

$$
\mathcal{T}(\omega)=\frac{4 n(\omega)}{[1+n(\omega)]^{2}\left[1-r^{2}(\omega)\right]} e^{i \omega(n(\omega)-1) L / c} .
$$

From Eqs (12) and (16) it is evident that the behavior of the function $S_{\mathrm{sq}}$ strongly depends on the behavior of the denominator $1-r^{2}(\omega)$.

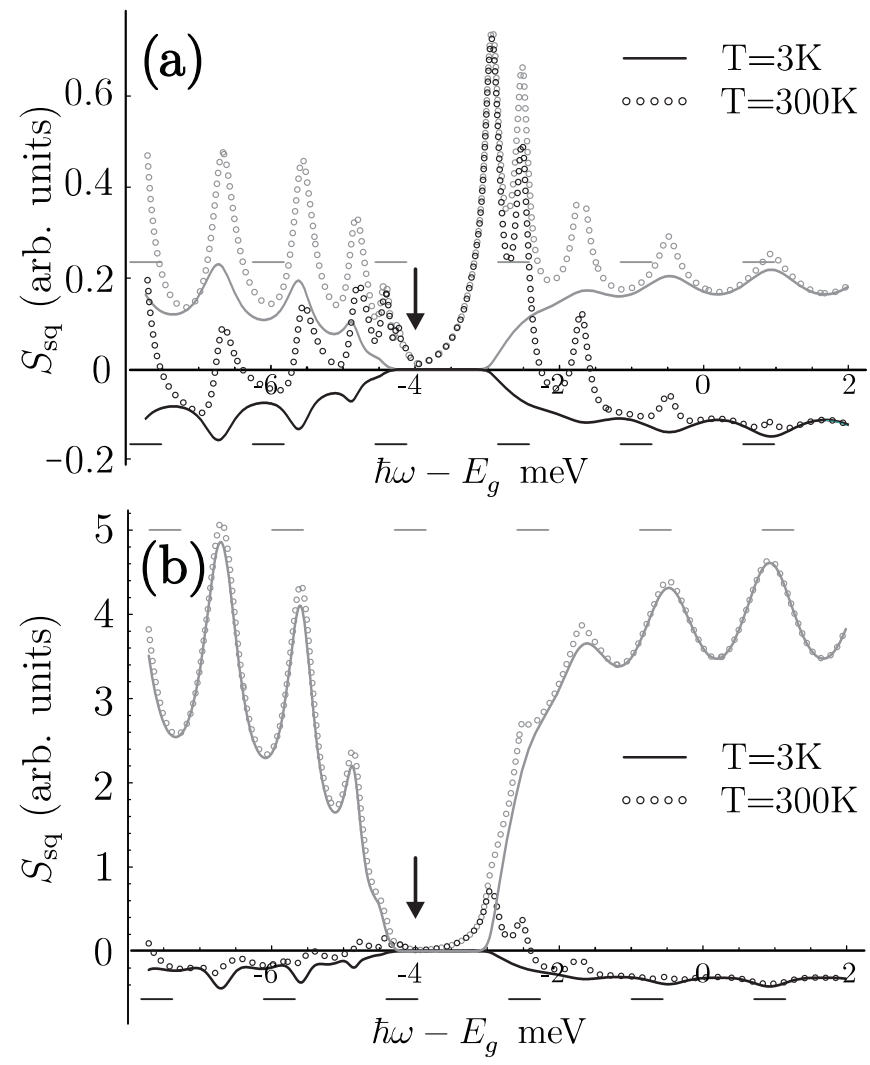

FIG. 1: Output squeezing spectrum for a GaAs slab $(L=$ $25 \mu \mathrm{m}$ ) near the $1 \mathrm{~s}$-excitonic resonance (indicated by an arrow) of bandwidth $=0.2 \mathrm{meV}$. The squeezing spectrum is shown for two temperatures and for $|\xi|=0.2$ (a), 1.2 (b). The dashed lines indicate the maximum and minimum noise level of the squeezed input field.

So, the squeezing spectrum shows an oscillating behavior with the maxima at the frequencies $\omega_{s}=\frac{2 \pi c}{L n^{\prime}(\omega)} s$ with integer $s$ (Fabry-Perot resonances).

In Fig. 1 1 we show the influence of a single excitonic resonance at $E_{x}=\hbar \omega_{0}$ on squeezed light propagating through a semiconductor slab, for different values of the squeezing strength $|\xi|$ where the Lorentz oscillator model has been used to simulate excitonic absorption [26]. The input (squeezed white noise) spectrum is depicted with dashed lines. The upper (gray) and lower (black) curves represent the maximum and the minimum noise level of the squeezing spectrum of the radiation after transmission through the slab. For high temperatures, the emission spectrum $I(\omega)$ plays a more pronounced role and it leads to a significant decrease of the nonclassical properties of the input field.

The squeezing spectrum for a highly excited semiconductor is shown in Fig. 2(a) for $|\xi|=0.2$. The susceptibility function has been calculated in the effective pairequation approximation [26] and is given in Fig. 2(b). In the output field, the squeezing is nearly destroyed, due to the dominance of the incoherent emission in the gain 

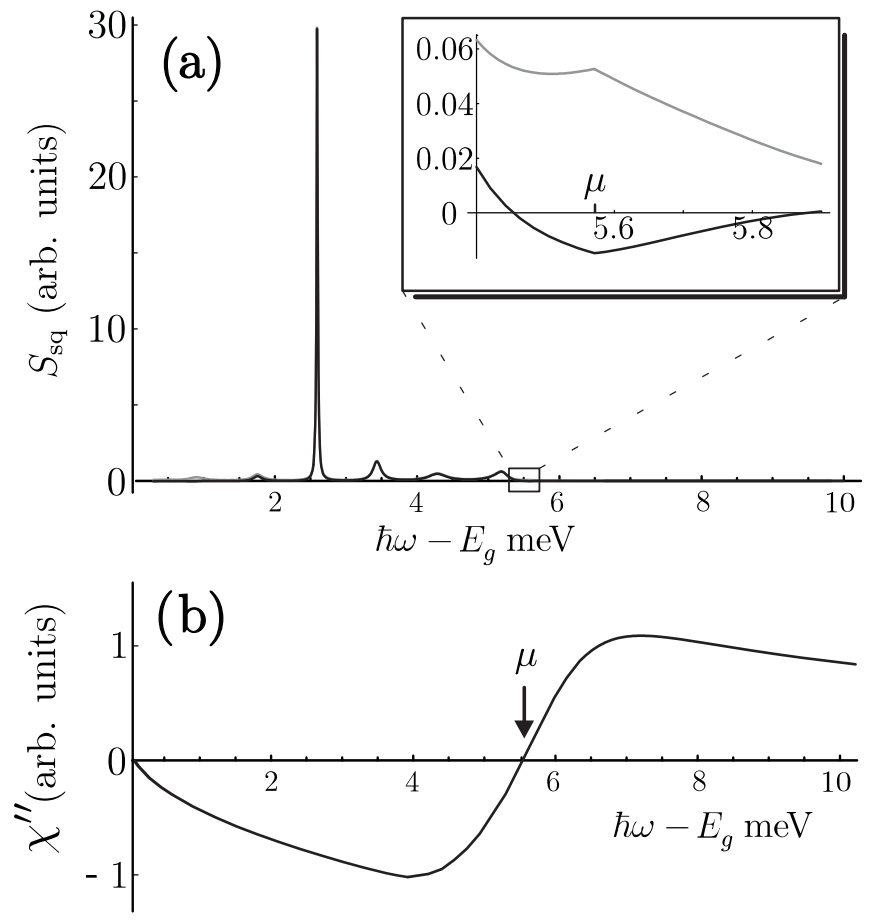

FIG. 2: Squeezing spectrum after transmission of a GaAs slab at temperature $\mathrm{T}=3 \mathrm{~K}$, pumped to a carrier density of $n=3 \cdot 10^{16} \mathrm{~cm}^{-3}$ (a) and the corresponding imaginary part of susceptibility (b). The input (dashed) and transmitted (solid) squeezing spectra are shown for $|\xi|=0.2$. An arrow indicates the chemical potential $\mu$ and the inset shows its vicinity.

region. Minor squeezing is only preserved in the vicinity of the crossover at $\hbar \omega=\mu$, where $\chi^{\prime \prime}=0$.

In conclusion, we have studied the relation between microscopic and macroscopic QED, leading to a clear physical interpretation of some basic quantities. By combining the input-output formalism of macroscopic QED with results of microscopic QED, the treatment of the propagation of nonclassical light in complex media becomes possible. As an example, we have studied squeezed-light propagation through a semiconductor slab.

This work was supported by the Deutsche Forschungsgemeinschaft, Sonderforschungsbereich 652 .

[1] D.-G. Welsch, W. Vogel, and T. Opatrny, in: Progress in Optics, Ed. E. Wolf, Vol. XXXIX, pp. 63 (1999).

[2] W. Vogel and D.-G. Welsch, Quantum Optics (WileyVCH, Weinheim, 2006).
[3] L. V. Keldysh, Zh. eksper. teor. fiz. 47, 1515 (1964).

[4] K. Henneberger and H. Haug, Phys. Rev. B 38, 9759 (1988).

[5] J. Kim, S. Somani and Y. Yamomoto, Nonclassical light from semiconductor lasers and LEDs (Springer, Berlin, 2001).

[6] P. Michler, A. Kiraz, C. Becher, W. Schoenfeld, P. Petroff, L. Zang, E. Hu, A. Imamoglu, Science 290, 2282 (2000).

[7] S. Strauf, P. Michler, M. Klude, D. Hommel, G. Bacher, A. Forchel, Phys. Rev. Lett. 89, 177403 (2002).

[8] W. Hoyer, M. Kira, S.W. Koch, H. Stolz, S. Mosor, J. Sweet, C. Ell, G. Khitrova, and H.M. Gibbs, Phys. Rev. Lett. 93, 067401 (2004).

[9] R.M. Stevenson, R.J. Young, P. Atkinson, K. Cooper, D.A. Ritchie, and A.J. Shields, Nature 439, 179 (2006).

[10] P.J. Young, R.M. Stevenson, P. Atkinson, K. Cooper, D.A. Ritchie, and A.J. Shields, New J. Phys. 8, 29 (2006).

[11] N. Akopian, N.H. Lindner, E. Poem, Y. Berlatzky, J. Avron, D. Gershoni, B.D. Gerardot and P.M. Petroff, Phys. Rev. Lett. 96, 130501 (2006).

[12] B. Huttner and S. M. Barnett, Phys. Rev. A 46, 4306 (1992)

[13] T. Gruner and D.-G. Welsch, Phys. Rev. A 53, 1818 (1996).

[14] R. Matloob, R. Loudon, M. Artoni, S. M. Barnett, and J. Jeffers, Phys. Rev. A 55, 1623 (1997).

[15] S. Scheel, L. Knöll, and D.-G. Welsch, Phys. Rev. A 58, 700 (1998).

[16] C. Raabe, S. Scheel, and D.-G. Welsch, Phys. Rev. A 75, 053813 (2007).

[17] C. Raabe and D.-G. Welsch, Eur. Phys. J., in press (arXiv:0710.2867v1 [quant-ph]).

[18] W. Schäfer and M. Wegener Semiconductor Optics and Transport Phenomena, (Springer, Berlin, 2002).

[19] K. Henneberger and S.W. Koch, Phys. Rev. Lett. 76, 1820 (1996).

[20] K. Henneberger and S.W. Koch in Quantum Theory of the Optical and Electronic Properties of Semiconductors, ed. S. W. Koch (World Scientific, Singapore, 1996).

[21] L. Knöll, S. Scheel, E. Schmidt, D.-G. Welsch, and A.V. Chizhov, Phys. Rev. A 59, 4716 (1999).

[22] K. Henneberger, arXiv:0710.5686 [condmat.str-el].

[23] B. Huttner, J.J. Baumberg and S.M. Barnett, Europhys. Lett. 16, 177 (1991).

[24] R. Kubo, J. Phys. Soc. Jpn. 12, 570 (1966); P.C. Martin and J. Schwinger, Phys. Rev. 115, 1432 (1959).

[25] R. Matloob, R. Loudon, S.M. Barnett, and J. Jeffers, Phys. Rev. A 52, 4823 (1995).

[26] H. Haug and S.W. Koch, Quantum theory of the optical and electronic properties of semiconductors (World Scientific, Singapore, 2005). 